\title{
CONDITIONAL CHARACTERISTIC FUNCTIONS OF MOLCHAN-GOLOSOV FRACTIONAL LÉVY PROCESSES WITH APPLICATION TO CREDIT RISK
}

\author{
HOLGER FINK, ${ }^{*}$ Technische Universität München
}

\begin{abstract}
Molchan-Golosov fractional Lévy processes (MG-FLPs) are introduced by way of a multivariate componentwise Molchan-Golosov transformation based on an $n$-dimensional driving Lévy process. Using results of fractional calculus and infinitely divisible distributions, we are able to calculate the conditional characteristic function of integrals driven by MG-FLPs. This leads to important predictions concerning multivariate fractional Brownian motion, fractional subordinators, and general fractional stochastic differential equations. Examples are the fractional Lévy Ornstein-Uhlenbeck and CoxIngersoll-Ross models. As an application we present a fractional credit model with a long range dependent hazard rate and calculate bond prices.

Keywords: Conditional characteristic function; macroeconomic variables process; longrange dependence; fractional Brownian motion; fractional Lévy process; prediction

2010 Mathematics Subject Classification: Primary 60G10; 60G22; 60G51; 60H10;

$60 \mathrm{H} 20 ; 91 \mathrm{G} 40$

Secondary 60G15; 91G30; 91G60
\end{abstract}

\section{Introduction}

In many financial or technical applications it is very useful to know the conditional characteristic function of certain stochastic processes because it is closely related to prediction; given the past evolution of a stochastic process, we are interested in its distribution at some time in the future. Consider the following example from the field of credit risk: given a short rate process $r=(r(v))_{v \in[0, T]}$ and a hazard rate process $\lambda=(\lambda(v))_{v \in[0, T]}$, with some market maturity date $T>0$, the price of a defaultable zero coupon bond at time $s$ with bond maturity $t, 0 \leq s \leq t \leq T$, is given by

$$
B(s, t)=\mathbf{1}_{\{\tau>s\}} \mathbb{E}^{\mathbb{Q}}\left[\mathrm{e}^{-\int_{s}^{t}(r(v)+\lambda(v)) \mathrm{d} v} \mid(r(v), \lambda(v)), v \in[0, s]\right],
$$

where the random time $\tau$ describes the default time of the bond and $\mathbb{Q}$ is some risk-neutral measure. To calculate this price it is useful to know the conditional characteristic function of the bivariate process $(r, \lambda)$.

Standard credit models describe $(r, \lambda)$ by an affine Markov process (see, e.g. [11] and [12]). Due to the Markov property, (1.1) can be calculated and takes a nice form which only depends on the random variable $(r(s), \lambda(s))$, i.e. today's level of the process $(r, \lambda)$. The past evolution of the path does not play any role. However, the ongoing financial crisis, which had its origin in the US credit market, showed that in real markets this assumption may be violated. In fact, the work

Received 12 July 2011; revision received 18 September 2012.

* Postal address: Center for Mathematical Sciences, Technische Universität München, Parkring 13, D-85748 Garching, Germany. Email address: fink@ma.tum.de 
of Henry and Zaffaroni [20] suggests that for many macroeconomic variables like interest and hazard rates, domestic gross products, supply and demand rates, and volatilities, there is strong empirical evidence against the Markov property. In particular, these processes show signs of long range dependence in their increments. This supports the use of fractional processes like fractional Brownian motion (fBm) and fractional Lévy processes for modeling the dynamics of $(r, \lambda)$ in $(1.1)$. However, the prediction problem becomes a lot more complicated since all past information will be involved in the price. The key is to note that these fractional processes can be described by kernel representations of affine Markov processes and, thus, certain structures remain.

This idea has been present in [7], where the authors focused, however, on contagion effects. Biagini, Fink and Klüppelberg [6] introduced a fractional Brownian credit market. Interest rate models driven by fBm were considered in [18] and also in [28]. However, all these approaches do not leave the Brownian framework.

In this paper we introduce the class of (multivariate) Molchan-Golosov fractional Lévy processes (MG-FLPs), including fractional Brownian motion and fractional subordinators (as defined in [5]), by way of a Molchan-Golosov transformation based on a general finite second-moment Lévy process. This idea has been proposed by Tikanmäki and Mishura [37], who, however, only considered the univariate case and used centered driving Lévy processes without Brownian parts, which basically excludes $\mathrm{fBm}$ and fractional subordinators. We then calculate the conditional characteristic functions of MG-FLP-related processes using fractional calculus and results on infinitely divisible distributions. Important examples like fractional Lévy Ornstein-Uhlenbeck processes (fLOUps) and Cox-Ingersoll-Ross processes (fLCIRps) are considered. The calculation of bond prices as in (1.1) is then straightforward.

Our paper is organized as follows. Section 2 presents preliminaries about Lévy processes, including integration and distributional results. In Section 3 we introduce MG-FLPs by way of an integral representation with respect to a multivariate Lévy process. Second order structure, path properties, and integration concepts are also considered. In Section 4 we present our main results on conditional characteristic functions of MG-FLP-related processes, including important examples like fLOUps and fLCIRps. As an application, in Section 5 we consider fractional credit models described by Vasicek dynamics and calculate bond prices. An example using a two-dimensional fractional Poisson subordinator, for $(r, \lambda)$, is presented.

We always assume that we are using a complete probability space $(\Omega, \mathcal{F}, \mathbb{P})$. We denote by $L^{2}(\Omega)$ the space of square integrable random variables. For a family of random variables $(X(i))_{i \in I}$, where $I$ is some index set, let $\sigma \overline{\{X(i), i \in I\}}$ denote the completion of the generated $\sigma$-algebra. For $A \subseteq \mathbb{R}$ and $n \in \mathbb{N}$, the spaces of integrable and square integrable functions $f: A \rightarrow \mathbb{R}^{n}$ are denoted by $L^{1}\left(A, \mathbb{R}^{n}\right)$ and $L^{2}\left(A, \mathbb{R}^{n}\right)$ respectively. In the case $n=1$ we just write $L^{1}(A)$ and $L^{2}(A)$. Furthermore, $\|\cdot\|$ is the $L^{2}$-norm and $\langle\cdot, \cdot\rangle$ the corresponding Euclidian scalar product. The positive and negative real half lines are denoted $\mathbb{R}_{+}$and $\mathbb{R}_{-}$ respectively. For a matrix $A, A^{\top}$ is the adjoint. The gamma function is denoted by $\Gamma$. Denote by $\mathbb{R}^{+}$the right halfline including zero.

\section{Preliminaries}

In the literature there are many different approaches given for defining fractional Lévy processes and a readable overview of two main concepts can be found in [37]. Both ways are mainly based on the idea of integrating memory into a Lévy process by choosing an appropriate kernel function. This can either be achieved by integration over the whole real line (see [25]) or on a compact interval (see [37]). However, these are not the only ways to obtain a generalization 
of fractional Brownian motion. For example, another possibility is to extend the harmonizable representation of $\mathrm{fBm}$ as presented in [2].

In this work we will choose the approach of Tikanmäki and Mishura [37] and generalize it to the multivariate case. Also, we will introduce a broader class of processes by using general finite second-moment Lévy processes. To avoid confusion with the other concepts we will call our class of processes Molchan-Golosov fractional Lévy processes (MG-FLPs).

Throughout the whole paper we will always consider a given multivariate Lévy process $\boldsymbol{L}=$ $(\boldsymbol{L}(t))_{t \in[0, T]}=\left(L^{1}(t), \ldots, L^{n}(t)\right)_{t \in[0, T]}^{\top}$, for $n \in \mathbb{N}$ and $T>0$, on a filtered probability space $\left(\Omega, \mathcal{F},\left(\mathcal{F}_{t}\right)_{t \in[0, T]}, \mathbb{P}\right)$ satisfying the usual conditions of right-continuity and completeness. The filtration (after possible augmentation) is assumed to be generated by $\boldsymbol{L}$ (cf. Theorem 2.1.9 of [1]). Then $\boldsymbol{L}$ can be described in terms of the characteristic triple $(\gamma, \Sigma, v)$ by its characteristic function $\mathbb{E}[\exp \{i\langle u, \boldsymbol{L}(t)\rangle\}]=\exp \{t \psi(u)\}, t \in[0, T]$, with

$$
\psi(u)=i\langle\gamma, u\rangle-\frac{1}{2} u^{\top} \Sigma u+\int_{\mathbb{R}^{n}}\left(\exp \{i\langle u, x\rangle\}-1-i\langle u, x\rangle \mathbf{1}_{\{\|x\|<1\}}\right) v(\mathrm{~d} x), \quad u \in \mathbb{R}^{n} .
$$

Here we have $\gamma \in \mathbb{R}^{n}, \Sigma \in \mathbb{R}^{n \times n}$ is symmetric and positive semidefinite, and the measure $v$ satisfies

$$
v(\{0\})=0 \quad \text { and } \quad \int_{\mathbb{R}^{n}}\left(\|x\|^{2} \wedge 1\right) v(\mathrm{~d} x)<\infty .
$$

Since we only consider finite second-moment Lévy processes, we also have that

$$
\int_{\mathbb{R}^{n}}\|x\|^{2} v(\mathrm{~d} x)<\infty
$$

Integration with respect to Lévy processes shall be understood in the usual $L^{2}(\Omega)$-sense (see, e.g. [31] or [36]). The following theorem will be crucial when predicting MG-FLPs. It is the multivariate version of Theorem 2.7 of [31] and it can be obtained using Proposition 2.17 of [36].

Theorem 2.1. For $f \in L^{2}\left([0, T], \mathbb{R}^{n \times n}\right)$ the integral $\int_{0}^{T} f(t) \mathrm{d} \boldsymbol{L}(t)$ exists as an $L^{2}(\Omega)$-limit of approximating step functions. Moreover, we have, for $u \in \mathbb{R}^{n}$,

$$
\mathbb{E}\left[\exp \left\{i\left\langle u, \int_{0}^{T} f(t) \mathrm{d} \boldsymbol{L}(t)\right\rangle\right\}\right]=\exp \left\{\int_{0}^{T} \psi\left(f(t)^{\top} u\right) \mathrm{d} t\right\} .
$$

Before stating the definition of MG-FLPs we need a few more concepts. First, define the fractional Riemann-Liouville integral with finite time horizon, for $d>0$, by

$$
\left(I_{T-}^{d} f\right)(s)=\frac{1}{\Gamma(d)} \int_{s}^{T} f(r)(r-s)^{d-1} \mathrm{~d} r, \quad 0 \leq s \leq T .
$$

For $f \in L^{2}(\mathbb{R})$, this always exists. We shall also need the fractional derivative with finite time horizon, for $d \in(0,1)$,

$$
\left(D_{T-}^{d} g\right)(u)=\frac{1}{\Gamma(1-d)}\left(\frac{g(u)}{(T-u)^{d}}+d \int_{u}^{T} \frac{g(u)-g(s)}{(s-u)^{d+1}} \mathrm{~d} s\right), \quad 0<u<T .
$$

The question of the existence of the fractional derivative is more sophisticated, for more details we refer the reader to [39]. For simplicity we shall only take fractional derivatives for suitable functions $g$ such that it always exists. 
We write $I_{T-}^{-d}=D_{T-}^{d}$. For $d=0$, we set $I_{T-}^{d}=D_{T-}^{d}=i d$.

We will also make use of the following spaces, introduced in [29] and [30]:

$$
\widetilde{\Lambda}_{T}^{d}:=\left\{\begin{array}{cll}
\{f:[0, T] \rightarrow \mathbb{R} \mid & \left.\int_{0}^{T}\left[s^{-d} I_{T-}^{d}\left((\cdot)^{d} f(\cdot)\right)(s)\right]^{2} \mathrm{~d} s<\infty\right\}, & d \in\left(0, \frac{1}{2}\right), \\
\{f:[0, T] \rightarrow \mathbb{R} \mid & \text { there exists } \phi_{f} \in L^{2}[0, T]: & \\
\left.f(s)=s^{-d} I_{T-}^{-d}\left((\cdot)^{d} \phi_{f}(\cdot)\right)(s)\right\}, & d \in\left(-\frac{1}{2}, 0\right) .
\end{array}\right.
$$

Because of our notation, for $d=0$, both sets are equal to $L^{2}[0, T]$. In light of Lemma 4.3 of [3], we shall adjust these spaces such that they are closed with respect to multiplication with an indicator function. Therefore, define, for $d \in\left(-\frac{1}{2}, \frac{1}{2}\right)$,

$$
\Lambda_{T}^{d}:=\left\{f:[0, T] \rightarrow \mathbb{R} \mid \text { for all }[s, t] \subseteq[0, T]: f \mathbf{1}_{[s, t]} \in \widetilde{\Lambda}_{T}^{d}\right\}
$$

For $d=(d(1), \ldots, d(n))^{\top} \in\left(-\frac{1}{2}, \frac{1}{2}\right)^{n}, n \in \mathbb{N}$, define

$$
\Lambda_{T}^{d}:=\left\{f:[0, T] \rightarrow \mathbb{R}^{n \times n} \mid f_{i j} \in \Lambda_{T}^{d(j)}, 1 \leq i, j \leq n\right\},
$$

where $f_{i j}$ denotes the $i j$ th component of $f$.

\section{Multivariate Molchan-Golosov fractional Lévy processes}

As already mentioned in Section 2, we will generalize the concept introduced by Tikanmäki and Mishura [37] to the multivariate case. Therefore, our processes will be defined by a compactly supported Molchan-Golosov transformation. However, in contrast to [37] we will also allow for a Brownian part which results in a fractional Brownian motion, and a noncentral driving Lévy process, possibly leading to a fractional subordinator introduced by Bender and Marquardt [5]. For $f \in \Lambda_{T}^{d}$ and $d=(d(1), \ldots, d(n))^{\top} \in\left(-\frac{1}{2}, \frac{1}{2}\right)^{n}, n \in \mathbb{N}$, define, for $s \in[0, T]$, the convolution operator

$$
\begin{aligned}
& z^{d}(f, s):= \\
& \left(\begin{array}{ccc}
c_{d(1)} s^{-d(1)} I_{T-}^{d(1)}\left((\cdot)^{d(1)} f_{11}(\cdot)\right)(s) & \cdots & c_{d(n)} s^{-d(n)} I_{T-}^{d(n)}\left((\cdot)^{d(n)}\right. \\
\left.f_{1 n}(\cdot)\right)(s) \\
\vdots & \ddots & \vdots \\
c_{d(1)} s^{-d(1)} I_{T-}^{d(1)}\left((\cdot)^{d(1)} f_{n 1}(\cdot)\right)(s) & \cdots & c_{d(n)} s^{-d(n)} I_{T-}^{d(n)}\left((\cdot)^{d(n)} f_{n n}(\cdot)\right)(s)
\end{array}\right)
\end{aligned}
$$

where, for $1 \leq j \leq n$,

$$
c_{d(j)}=\left(\frac{(2 d(j)+1) \Gamma(d(j)+1) \Gamma(1-d(j))}{\Gamma(1-2 d(j))}\right)^{1 / 2} .
$$

The very general definition of MG-FLPs follows.

Definition 3.1. For $d=(d(1), \ldots, d(n))^{\top} \in\left(-\frac{1}{2}, \frac{1}{2}\right)^{n}, n \in \mathbb{N}$, we define the kernel function $z^{d}\left(\hat{\mathbf{1}}_{[0, \cdot)}, \cdot\right):[0, T] \times[0, T] \rightarrow \mathbb{R}^{n \times n}$ using, for $s, t \in[0, T]$,

$$
\hat{\mathbf{1}}_{[0, t]}(s):=\left(\begin{array}{ccc}
\mathbf{1}_{[0, t]}(s) & \cdots & 0 \\
\vdots & \ddots & \vdots \\
0 & \cdots & \mathbf{1}_{[0, t]}(s)
\end{array}\right) .
$$


Then a Molchan-Golosov fractional Lévy process (MG-FLP)

$$
\boldsymbol{L}^{d}=\left(\boldsymbol{L}^{d}(t)\right)_{t \in[0, T]}=\left(L^{d(1)}(t), \ldots, L^{d(n)}(t)\right)_{t \in[0, T]}^{\top}
$$

is defined by

$$
\boldsymbol{L}^{d}(t)=\int_{0}^{t} z^{d}\left(\hat{\mathbf{1}}_{[0, t]}, s\right) \mathrm{d} \boldsymbol{L}(s), \quad t \in[0, T] .
$$

Remark 3.1. The integral in (3.1) can be considered in the $L^{2}(\Omega)$-sense or in the pathwise Riemann-Stieltjes-sense. The first assertion is clear by [31] and by the fact that the kernel is square-integrable. The second one follows because, as a finite second-moment Lévy process, $\boldsymbol{L}$ is of bounded $p$-variation for all $p>2$ (cf. Theorem 2 of [27], based on the BlumenthalGetoor-index introduced in [9]), and $z^{d}(t, s)$, on $(0, t)$, is componentwise of bounded variation in the variable $s$; cf. [38].

Using the Lévy-Itô decomposition we obtain

$$
\begin{aligned}
\boldsymbol{L}(t) & =\mathbb{E}[\boldsymbol{L}(t)]+(\boldsymbol{L}(t)-\mathbb{E}[\boldsymbol{L}(t)]) \\
& =\mathbb{E}[\boldsymbol{L}(1)] \cdot t+\boldsymbol{B}(t)+\boldsymbol{S}(t), \quad t \in[0, T],
\end{aligned}
$$

where $\boldsymbol{B}$ is an $n$-dimensional Brownian motion with $\boldsymbol{B}(1) \sim N(0, \Sigma)$ and $\boldsymbol{S}$ is a zero-mean Lévy process without Brownian part. Furthermore $\boldsymbol{B}$ and $\boldsymbol{S}$ are independent. This leads to the MG-FLP decomposition

$$
\boldsymbol{L}^{d}(t)=\int_{0}^{t} z^{d}\left(\hat{\mathbf{1}}_{[0, t]}, s\right) \mathrm{d} s \cdot \mathbb{E}[\boldsymbol{L}(1)]+\boldsymbol{B}^{d}(t)+\boldsymbol{S}^{d}(t), \quad t \in[0, T],
$$

where the first integral is understood componentwise. Further, $\boldsymbol{B}^{d}$ and $\boldsymbol{S}^{d}$ are defined as in (3.1), i.e. they are a multivariate $\mathrm{fBm}$ and a zero-mean MG-FLP.

Example 3.1. Let $n=1$ in Definition 3.1.

(i) Choosing as the driving Lévy process a standard Brownian motion, we get a classical fBm on $[0, T]$; cf. [34].

(ii) Taking a strictly increasing subordinator as the driving Lévy process leads to a fractional subordinator in the sense of Example 1 of [5]. In particular, the resulting MG-FLP is almost surely (a.s.) increasing.

The next results follow by standard properties of Lévy processes; see, e.g. [24], [31], and [35]. A brief look at the autocovariance of an MG-FLP leads to the following proposition.

Proposition 3.1. For $s, t \in[0, T]$, we have, for the mean-value and the autocovariance function,

(i) $\mathbb{E}\left[\boldsymbol{L}^{d}(t)\right]=\int_{0}^{t} z^{d}\left(\hat{\mathbf{1}}_{[0, t]}, s\right) \mathrm{d} s \cdot \mathbb{E}[\boldsymbol{L}(1)]$,

$$
\begin{aligned}
\operatorname{cov}\left[\boldsymbol{L}^{d}(t), \boldsymbol{L}^{d}(s)\right] & \\
=\frac{1}{2}\left(c _ { d ( i ) , d ( j ) } \operatorname { c o v } [ L ^ { i } ( 1 ) , L ^ { j } ( 1 ) ] \left(t^{d(i)+d(j)+1}+s^{d(i)+d(j)+1}\right.\right. & \left.\left.-|t-s|^{d(i)+d(j)+1}\right)\right)_{1 \leq i, j \leq n}
\end{aligned}
$$


where

$$
c_{d(i), d(j)}=\frac{\sqrt{\Gamma(2 d(i)+2) \sin \left(\pi\left(d(i)+\frac{1}{2}\right)\right)} \sqrt{\Gamma(2 d(j)+2) \sin \left(\pi\left(d(j)+\frac{1}{2}\right)\right)}}{\Gamma(d(i)+d(j)+2) \sin (\pi(d(i)+d(j)+1) / 2)} .
$$

Proof. The first part is clear from the decomposition (3.2). The second one follows by

$$
\begin{aligned}
\operatorname{cov}\left[\boldsymbol{L}^{d}(t), \boldsymbol{L}^{d}(s)\right] & =\int_{0}^{T} z^{d}\left(\hat{\mathbf{1}}_{[0, t]}, u\right) \operatorname{cov}[\boldsymbol{L}(t), \boldsymbol{L}(s)]\left(z^{d}\left(\hat{\mathbf{1}}_{[0, s]}, u\right)\right)^{\top} \mathrm{d} u \\
& =\left(\operatorname{cov}\left[L^{i}(t), L^{j}(s)\right] \int_{0}^{T} z_{i i}^{d}\left(\hat{\mathbf{1}}_{[0, t]}, u\right) z_{j j}^{d}\left(\hat{\mathbf{1}}_{[0, s]}, u\right) \mathrm{d} u\right)_{1 \leq i, j \leq n} .
\end{aligned}
$$

The calculation of the last integrals is similar to Equation (2.17) of [14].

Remark 3.2. Proposition 3.1 (ii) shows that MG-FLPs have the same second-order structure as $\mathrm{fBm}$ (up to a constant) and, therefore, it is clear that, for all $1 \leq i \leq n$, the increments of the univariate process $L^{d(i)}$ exhibit a similar memory structure as in the case of $\mathrm{fBm}$.

The next lemma summarizes the main properties of MG-FLPs and is the multivariate extension of Proposition 3.7 of [37].

Lemma 3.1. We have, for $d=(d(1), \ldots, d(n))^{\top}$, the following properties.

(i) An MG-FLP without a Gaussian component has a.s. continuous paths if and only if $d \in\left(0, \frac{1}{2}\right)^{n}$.

(ii) An MG-FLP without a Gaussian component has a.s. Hölder continuous paths of any order $\alpha<\min [d]$ if and only if $d \in\left(0, \frac{1}{2}\right)^{n}$.

(iii) If $d(i) \in\left(-\frac{1}{2}, 0\right)$, for some $1 \leq i \leq n$, then the MG-FLP has discontinuous and unbounded sample paths with positive probability.

Before coming to conditional characteristic functions of MG-FLPs we need to define integration. This will be done by using the usual approach for $L^{2}(\Omega)$; see, e.g. [25], [29], [30], or [37].

Consider simple functions of the form

$$
f(\cdot)=\sum_{k=1}^{m} a_{k} \mathbf{1}_{\left[t_{k}, t_{k+1}\right)}(\cdot),
$$

where $m \in \mathbb{N}, m \geq 1,0 \leq t_{1} \leq \cdots \leq t_{m} \leq T$, and $a_{k} \in \mathbb{R}^{n \times n}$ for $1 \leq k \leq m$. Then we define

$$
\begin{aligned}
\int_{0}^{T} f(s) \mathrm{d} \boldsymbol{L}^{d}(s) & :=\sum_{k=1}^{m} a_{k}\left(\boldsymbol{L}^{d}\left(t_{k+1}\right)-\boldsymbol{L}^{d}\left(t_{k}\right)\right) \\
& =\sum_{k=1}^{m}\left(\begin{array}{c}
\sum_{j=1}^{n}\left(a_{k}\right)_{1 j}\left(L^{d(j)}\left(t_{k+1}\right)-L^{d(j)}\left(t_{k}\right)\right) \\
\vdots \\
\sum_{j=1}^{n}\left(a_{k}\right)_{n j}\left(L^{d(j)}\left(t_{k+1}\right)-L^{d(j)}\left(t_{k}\right)\right)
\end{array}\right) .
\end{aligned}
$$

A simple calculation leads to

$$
\int_{0}^{T} f(s) \mathrm{d} \boldsymbol{L}^{d}(s)=\int_{0}^{T} z^{d}(f, s) \mathrm{d} \boldsymbol{L}(s)
$$


Now we obtain, from the definition of $\Lambda_{T}^{d}$ and Theorem 2.1, the following theorem.

Theorem 3.1. For $d=(d(1), \ldots, d(n))^{\top} \in\left(-\frac{1}{2}, \frac{1}{2}\right)^{n}, n \in \mathbb{N}$, let $f \in \Lambda_{T}^{d}$. Then the integral $\int_{0}^{T} f(s) \mathrm{d} \boldsymbol{L}^{d}(s)$ exists as a (componentwise) $L^{2}(\Omega)$-limit of approximating step functions in $\Lambda_{T}^{d}$ (also componentwise). Furthermore, we have the identity

$$
\int_{0}^{T} f(s) \mathrm{d} \boldsymbol{L}^{d}(s)=\int_{0}^{T} z^{d}(f, s) \mathrm{d} \boldsymbol{L}(s)
$$

which holds (componentwise) in $L^{2}(\Omega)$.

Furthermore, we find some distributional results on MG-FLP driven integrals. The proof uses Theorem 2.1, Theorem 3.1, and the fact that $z^{d}(t, s)$ is Hermitian and symmetric for $s, t \in[0, T]$.

Theorem 3.2. For $d=(d(1), \ldots, d(n))^{\top} \in\left(-\frac{1}{2}, \frac{1}{2}\right)^{n}, n \in \mathbb{N}$, let $f \in \Lambda_{T}^{d}$. Then we have, for all $u \in \mathbb{R}^{n}$,

$$
\begin{aligned}
\mathbb{E}\left[\exp \left\{i\left\langle u, \int_{0}^{T} f(s) \mathrm{d} \boldsymbol{L}^{d}(s)\right\rangle\right\}\right] \\
=\exp \left\{\int_{0}^{T} \psi\left(z^{d}(f, s)^{\top} u\right) \mathrm{d} s\right\} \\
=\exp \left\{i \int_{0}^{T}\left\langle z^{d}(f, s) \gamma, u\right\rangle \mathrm{d} s-\frac{1}{2} \int_{0}^{T} u^{\top} z^{d}(f, s) \Sigma z^{d}(f, s)^{\top} u \mathrm{~d} s\right. \\
\left.\quad+\int_{0}^{T} \int_{\mathbb{R}^{n}}\left(\mathrm{e}^{i\left\langle u, z^{d}(f, s) x\right\rangle}-1-i\left\langle u, z^{d}(f, s) x\right\rangle \mathbf{1}_{\left\{\left\|z^{d}(f, s) x\right\|<1\right\}}\right) v(\mathrm{~d} x) \mathrm{d} s\right\} .
\end{aligned}
$$

The characteristic function of an MG-FLP is calculated in Example 3.2.

Example 3.2. For each fixed $t \in[0, T]$ the random vector $\boldsymbol{L}^{d}(t)$ is infinitely divisible and its characteristic function is, for $u \in \mathbb{R}^{n}$, given by

$$
\begin{aligned}
\mathbb{E}\left[\exp \left\{i\left\langle u, \boldsymbol{L}^{d}(t)\right\rangle\right\}\right] \\
=\exp \left\{\int_{0}^{T} \psi\left(z^{d}(t, s) u\right) \mathrm{d} s\right\} \\
=\exp \left\{i \int_{0}^{T}\left\langle z^{d}(t, s) \gamma, u\right\rangle \mathrm{d} s-\frac{1}{2} \int_{0}^{T} u^{\top} z^{d}(t, s) \Sigma z^{d}(t, s) u \mathrm{~d} s\right. \\
\left.\quad+\int_{0}^{T} \int_{\mathbb{R}^{n}}\left(\mathrm{e}^{i\left\langle u, z^{d}(t, s) x\right\rangle}-1-i\left\langle u, z^{d}(t, s) x\right\rangle \mathbf{1}_{\left\{\left\|z^{d}(t, s) x\right\|<1\right\}}\right) v(\mathrm{~d} x) \mathrm{d} s\right\} .
\end{aligned}
$$

Remark 3.3. When $d \in\left(0, \frac{1}{2}\right)^{n}$, it is also possible to define pathwise integration with respect to MG-FLPs using Hölder continuity, as used in [10], or a $p$-variation approach; see [16]. Another possible approach would be via a Skorohod-type integral using the $S$-transform as suggested by Bender and Marquardt [4]. 


\section{Results on conditional characteristic functions}

In this section we state and prove our main theorems about the conditional characteristic functions of MG-FLP driven integrals and related processes. Define, for $d \in\left(-\frac{1}{2}, \frac{1}{2}\right)$ and suitable $f:[0, T] \rightarrow \mathbb{R}^{n \times n}$, the deconvolution operator

$$
\begin{aligned}
& z_{\star}^{d}(f, s):= \\
& \left(\begin{array}{cccc}
c_{-d(1)}^{-1} s^{d(1)} I_{T-}^{d(1)}\left((\cdot)^{-d(1)} f_{11}(\cdot)\right)(s) & \cdots & c_{-d(n)}^{-1} s^{d(n)} I_{T-}^{d(n)}\left((\cdot)^{-d(n)} f_{1 n}(\cdot)\right)(s) \\
\vdots & \ddots & \vdots \\
c_{-d(1)}^{-1} s^{d(1)} I_{T-}^{d(1)}\left((\cdot)^{-d(1)} f_{n 1}(\cdot)\right)(s) & \cdots & c_{-d(n)}^{-1} s^{d(n)} I_{T-}^{d(n)}\left((\cdot)^{-d(n)} f_{n n}(\cdot)\right)(s)
\end{array}\right) .
\end{aligned}
$$

First, we need a technical lemma which will be crucial to derive the prediction formula and ensure that all appearing deconvolution operators exist.

Lemma 4.1. For $d=(d(1), \ldots, d(n))^{\top} \in\left(-\frac{1}{2}, \frac{1}{2}\right)^{n}, n \in \mathbb{N}$, let $f \in \Lambda_{T}^{d}$. Then the following assertions hold for all $0 \leq s \leq t \leq T$.

(i) For all $1 \leq i, j \leq n$, we have $z_{i j}^{d}\left(f \mathbf{1}_{[s, t]}, \cdot\right) \in L^{2}([0, T]) \subset L^{1}([0, T])$.

(ii) The function

$$
[0, s] \rightarrow \mathbb{R}^{n \times n}, \quad v \mapsto z_{\star}^{-d}\left(\mathbf{1}_{[0, s]} z^{d}\left(f \mathbf{1}_{[s, t]}, \cdot\right), v\right)
$$

exists componentwise and belongs to $\widetilde{\Lambda}_{s}^{d}$.

(iii) For all $v \in[0, T]$,

$$
z^{d}\left(z_{\star}^{-d}\left(\mathbf{1}_{[0, s]} z^{d}\left(f \mathbf{1}_{[s, t]}, \cdot\right), \cdot\right), v\right)=\mathbf{1}_{[0, s]}(v) z^{d}\left(f \mathbf{1}_{[s, t]}, v\right) .
$$

Proof. Since $f \in \Lambda_{T}^{d}$ it follows that $f \mathbf{1}_{[s, t]} \in \Lambda_{T}^{d}$. By the definition of $\Lambda_{T}^{d}$, we therefore have $z_{i j}^{d}\left(f \mathbf{1}_{[s, t]}, \cdot\right) \in L^{2}([0, T]) \subset L^{1}([0, T])$, which leads to (i). The existence of the function in assertion (ii) can be obtained by using a similar approximation argument as used in the proof of Lemma 1 of [13]. The second statement in (ii) and (iii) follow by the definition of $\widetilde{\Lambda}_{s}^{d}$ and by applying Theorem 2.5 of [33] componentwise.

Remark 4.1. Clearly, $\boldsymbol{L}^{d}$ is adapted to the filtration $\left(\mathcal{F}_{t}\right)_{t \in[0, T]}$ which can now be directly seen by the deconvolution formula (cf. [22])

$$
\boldsymbol{L}(t)=\int_{0}^{t} z_{\star}^{-d}\left(\hat{\mathbf{1}}_{[0, t]}, s\right) \mathrm{d} \boldsymbol{L}^{d}(s), \quad t \in[0, T] .
$$

Furthermore, we remark that this is not the case for fractional Lévy processes as defined in [25]. However, in contrast to the processes in [25], MG-FLPs do not have stationary increments in general; cf. Proposition 3.11 of [37].

Theorem 4.1. For $d=(d(1), \ldots, d(n))^{\top} \in\left(-\frac{1}{2}, \frac{1}{2}\right)^{n}, n \in \mathbb{N}$, let $f \in \Lambda_{T}^{d}$ and

$$
\mathcal{F}_{t}=\sigma \overline{\left\{\int_{0}^{s} f(v) \mathrm{d} \boldsymbol{L}^{d}(v), s \in[0, t]\right\}}, \quad t \in[0, T] .
$$


Then we have, for all $0 \leq s \leq t \leq T$ and $u \in \mathbb{R}^{n}$,

$$
\begin{aligned}
\mathbb{E}\left[\exp \left\{i\left\langle u, \int_{0}^{t} f(v) \mathrm{d} \boldsymbol{L}^{d}(v)\right\rangle \mid \mathcal{F}_{s}\right\}\right] \\
=\exp \left\{i\left\langle u, \int_{0}^{s} f(v) \mathrm{d} \boldsymbol{L}^{d}(v)+\int_{0}^{s} z_{\star}^{-d}\left(\mathbf{1}_{[0, s]} z^{d}\left(f \mathbf{1}_{[s, t]}, \cdot\right), v\right) \mathrm{d} \boldsymbol{L}^{d}(v)\right\rangle\right. \\
\left.+\int_{s}^{t} \psi\left(z^{d}\left(f \mathbf{1}_{[s, t]}, v\right)^{\top} u\right) \mathrm{d} v\right\} .
\end{aligned}
$$

Proof. Recall that an MG-FLP is adapted to the filtration $\left(\mathcal{F}_{t}\right)_{t \in[0, T]}$ generated by the corresponding Lévy process. Since the random variable $\int_{0}^{t} f(v) d \boldsymbol{L}^{d}(v)$ is $\widetilde{F}_{s}$-measurable, it is enough to consider the conditional characteristic function of $\int_{s}^{t} f(v) \mathrm{d} \boldsymbol{L}^{d}(v)$. Applying Theorem 3.1, we switch from the MG-FLP to the corresponding Lévy process and obtain

$$
\begin{aligned}
\int_{s}^{t} f(v) \mathrm{d} \boldsymbol{L}^{d}(v) & =\int_{0}^{T} z^{d}\left(f \mathbf{1}_{[s, t]}, v\right) \mathrm{d} \boldsymbol{L}(v) \\
& =\int_{0}^{s} z^{d}\left(f \mathbf{1}_{[s, t]}, v\right) \mathrm{d} \boldsymbol{L}(v)+\int_{s}^{T} z^{d}\left(f \mathbf{1}_{[s, t]}, v\right) \mathrm{d} \boldsymbol{L}(v) .
\end{aligned}
$$

The first summand on the right-hand side is again $\mathcal{F}_{S}$-measurable and, therefore, we obtain

$$
\begin{aligned}
\mathbb{E}\left[\exp \left\{i\left\langle u, \int_{0}^{t} f(v) \mathrm{d} \boldsymbol{L}^{d}(v)\right\rangle \mid \mathcal{F}_{s}\right\}\right] \\
=\exp \left\{i\left\langle u, \int_{0}^{s} f(v) \mathrm{d} \boldsymbol{L}^{d}(v)+\int_{0}^{s} z^{d}\left(f \mathbf{1}_{[s, t]}, v\right) \mathrm{d} \boldsymbol{L}(v)\right\rangle\right\} \\
\times \mathbb{E}\left[\exp \left\{i\left\langle u, \int_{s}^{T} z^{d}\left(f \mathbf{1}_{[s, t]}, v\right) \mathrm{d} \boldsymbol{L}(v)\right\rangle \mid \mathcal{F}_{s}\right\}\right] .
\end{aligned}
$$

However, due to independent increments of Lévy processes, we have

$$
\mathbb{E}\left[\exp \left\{i\left\langle u, \int_{s}^{T} z^{d}\left(f \mathbf{1}_{[s, t]}, v\right) \mathrm{d} \boldsymbol{L}(v)\right\rangle \mid \mathcal{F}_{s}\right\}\right]=\mathbb{E}\left[\exp \left\{i\left\langle u, \int_{s}^{T} z^{d}\left(f \mathbf{1}_{[s, t]}, v\right) \mathrm{d} \boldsymbol{L}(v)\right\rangle\right\}\right],
$$

and applying Theorem 2.1 leads to

$$
\mathbb{E}\left[\exp \left\{i\left\langle u, \int_{s}^{T} z^{d}\left(f \mathbf{1}_{[s, t]}, v\right) \mathrm{d} \boldsymbol{L}(v)\right\rangle\right\}\right]=\exp \left\{\int_{s}^{t} \psi\left(z^{d}\left(f \mathbf{1}_{[s, t]}, v\right)^{\top} u\right) \mathrm{d} v\right\},
$$

where we have used the fact that $z^{d}\left(f \mathbf{1}_{[s, t]}, v\right)=0$ if $v \in[t, T]$. Since we want the prediction formula in terms of the MG-FLP and not the driving Lévy process, we invoke Lemma 4.1 (ii) and apply Theorem 3.1 again to obtain

$$
\int_{0}^{s} z^{d}\left(f \mathbf{1}_{[s, t]}, v\right) \mathrm{d} \boldsymbol{L}(v)=\int_{0}^{s} z_{\star}^{-d}\left(\mathbf{1}_{[0, s]} z^{d}\left(f \mathbf{1}_{[s, t]}, \cdot\right), v\right) \mathrm{d} \boldsymbol{L}^{d}(v) .
$$

Putting everything together we arrive at the assertion.

Remark 4.2. Every $f \in \Lambda_{T}^{d}$, with $f_{i j}(u) \neq 0$ for all $u \in[0, T]$ and $1 \leq i, j \leq n$, satisfies the conditions of Theorem 4.1. This follows since an MG-FLP is adapted to the natural filtration of the driving Lévy process; cf. Remark 3.2 of [37]. 
Example 4.1. (Univariate $f B m$.) In Theorem 4.1, choose $n=1, f=\mathbf{1}_{[0, t]}, 0 \leq t \leq T$, and take as the driving Lévy process a standard Brownian motion, i.e. $\boldsymbol{L}=B$. Then $\boldsymbol{L}^{d}=B^{d}$ is a univariate $\mathrm{fBm}$. Using Theorem 3.1 and Remark 3.2 of [18], the conditional characteristic function is, for $0 \leq s \leq t \leq T$, given by

$$
\begin{aligned}
& \mathbb{E}\left[\exp \left\{i u B^{d}(t) \mid \mathcal{F}_{s}\right\}\right] \\
& =\exp \left\{i u\left[B^{d}(s)+\int_{0}^{s} \Psi^{d}(s, t, v) \mathrm{d} B^{d}(v)\right]\right. \\
& \left.\quad-\frac{u^{2}}{2}\left[\left\|\mathbf{1}_{[s, t]}\right\|_{d, T}^{2}-\left\|\Psi^{d}(s, t, \cdot) \mathbf{1}_{[0, s]}\right\|_{d, T}^{2}\right]\right\}
\end{aligned}
$$

where

$$
\Psi^{d}(s, t, v)=v^{-d}\left(I_{s-}^{-d}\left(I_{t-}^{d}(\cdot)^{d} \mathbf{1}_{[s, t]}(\cdot)\right)\right)(v), \quad v \in(0, s)
$$

and $\|\cdot\|_{d, T}^{2}$ is as defined in Section 2 of [18]. This matches the result of Theorem 4.1 since $I_{t-}^{-d} \mathbf{1}_{[0, s]}(\cdot) f(\cdot)=I_{s-}^{-d} f(\cdot)$ in the situation above. Furthermore, we have

$$
\left\|\mathbf{1}_{[s, t]}\right\|_{d, T}^{2}-\left\|\Psi^{d}(s, t, \cdot) \mathbf{1}_{[0, s]}\right\|_{d, T}^{2}=\left\|z^{d}\left(\mathbf{1}_{[s, t]}, \cdot\right) \mathbf{1}_{[s, t]}(\cdot)\right\|^{2} .
$$

Example 4.2. (Univariate gamma MG-FLP.) In Theorem 4.1, choose $n=1, f=\mathbf{1}_{[0, t]}, 0 \leq$ $t \leq T$, and take as the driving Lévy process a univariate gamma process $G=(G(t))_{t \in[0, T]}$. Its distribution is then characterized by

$$
\psi(u)=-\gamma \log \left(1-\frac{u}{\lambda}\right) \mathbf{1}_{[0, \lambda)}(u),
$$

with $\gamma, \lambda>0$. By Theorem 4.1 we obtain, for the gamma MG-FLP $G^{d}=\left(G^{d}(t)\right)_{t \in[0, T]}$ and $0 \leq s \leq t \leq T$,

$$
\begin{aligned}
\mathbb{E}\left[\exp \left\{i u G^{d}(t) \mid \mathcal{F}_{s}\right\}\right] \\
=\exp \left\{i u\left[G^{d}(s)+\int_{0}^{s} z_{\star}^{-d}\left(\mathbf{1}_{[0, s]} z^{d}\left(\mathbf{1}_{[s, t]}, \cdot\right), v\right) \mathrm{d} G^{d}(v)\right]\right\} \\
\quad \times \exp \left\{\gamma \log (\lambda)[t-s]-\gamma \int_{s}^{t} \log \left(\lambda-z^{d}\left(\mathbf{1}_{[s, t]}, v\right) u\right) \mathrm{d} v\right\} .
\end{aligned}
$$

Example 4.3. (Bivariate Poisson MG-FLP.) In Theorem 4.1, choose $n=2, f=\mathbf{1}_{[0, t]}, 0 \leq$ $t \leq T$, and take as the driving Lévy process a bivariate Poisson process, i.e. here we take independent Poisson processes $Z_{i}$, on $[0, T]$, with intensities $\eta_{i} \geq 0$ for $i=1,2,3$ and define

$$
\boldsymbol{L}:=\left(Z_{1}+Z_{2}, Z_{2}+Z_{3}\right)^{\top}
$$

The distribution of this bivariate Lévy process is then characterized by

$$
\begin{aligned}
\psi(u) & =\int_{\mathbb{R}^{2}}\left(\exp \{i\langle u, x\rangle\}-1-i\langle u, x\rangle \mathbf{1}_{\{\|x\|<1\}}\right) v(\mathrm{~d} x) \\
& =\int_{\mathbb{R}^{2}}(\exp \{i\langle u, x\rangle\}-1) v(\mathrm{~d} x), \quad u \in \mathbb{R}^{2}
\end{aligned}
$$


with $v(\mathrm{~d} x)=\eta_{1} \delta_{\{1\} \times\{0\}}(\mathrm{d} x)+\eta_{2} \delta_{\{1\} \times\{1\}}(\mathrm{d} x)+\eta_{3} \delta_{\{0\} \times\{1\}}(\mathrm{d} x)$. Theorem 4.1 leads now, for $0 \leq s \leq t \leq T$, to

$$
\begin{aligned}
\mathbb{E}\left[\exp \left\{i\left\langle u, \boldsymbol{L}^{d}(t)\right\rangle \mid \mathcal{F}_{s}\right\}\right] \\
=\exp \left\{i\left\langle u, \boldsymbol{L}^{d}(s)+\int_{0}^{s} z_{\star}^{-d}\left(\mathbf{1}_{[0, s]} z^{d}\left(\mathbf{1}_{[s, t]}, \cdot\right), v\right) \mathrm{d} \boldsymbol{L}^{d}(v)\right\rangle\right\} \\
\times \exp \left\{\eta_{1} \int_{s}^{t}\left(\exp \left(i \sum_{j=1}^{2} z_{1 j}^{d}\left(\mathbf{1}_{[s, t)}, v\right) u_{j}\right)-1\right) \mathrm{d} v\right\} \\
\times \exp \left\{\eta_{2} \int_{s}^{t}\left(\exp \left(\sum_{k=1}^{2} \sum_{j=1}^{2} z_{k j}^{d}\left(\mathbf{1}_{[s, t)}, v\right) u_{j}\right)-1\right) \mathrm{d} v\right\} \\
\quad \times \exp \left\{\eta_{3} \int_{s}^{t}\left(\exp \left(i \sum_{j=1}^{2} z_{2 j}^{d}\left(\mathbf{1}_{[s, t)}, v\right) u_{j}\right)-1\right) \mathrm{d} v\right\} .
\end{aligned}
$$

Next, we consider MG-FLP-driven Ornstein-Uhlenbeck processes, starting with the definition. Similar processes were considered by Marquardt [26] and Klüppelberg and Matsui [23]. However, in contrast to our work, they define their underlying fractional Lévy processes by an integral representation over the whole real line as in [25]. Postponing the usual question of existence and uniqueness until Proposition 4.1, we use the following definition.

Definition 4.1. For $d=(d(1), \ldots, d(n))^{\top} \in\left(0, \frac{1}{2}\right)^{n}$, take $\sigma \in \Lambda_{T}^{d}, k:[0, T] \rightarrow \mathbb{R}^{n}$, and $a:[0, T] \rightarrow \mathbb{R}^{n \times n}$, where $k$ and $a$ are componentwise locally integrable such that

$$
\mathrm{e}^{-\int_{\cdot}^{t} a(v) \mathrm{d} v} \sigma(\cdot) \in \Lambda_{T}^{d}
$$

for all $t \in[0, T]$. Then the (unique) solution to the stochastic differential equation (SDE),

$$
\mathrm{d} \mathfrak{L}^{d}(t)=\left(k(t)-a(t) \mathfrak{L}^{d}(t)\right) \mathrm{d} t+\sigma(t) \mathrm{d} \boldsymbol{L}^{d}(t), \quad t \in[0, T], \quad \mathfrak{L}^{d}(0) \in \mathbb{R}^{n},
$$

is called a fractional Lévy Ornstein-Uhlenbeck process (fLOUp).

The next proposition ensures the existence of a solution. Its uniqueness follows by a simple application of Gronwall's Lemma (e.g. Theorem 3.1 of [21]) similar to the classical Brownian case.

Proposition 4.1. In the situation of Definition 4.1 we have, for $t \in[0, T]$,

$$
\mathfrak{L}^{d}(t)=\mathrm{e}^{-\int_{0}^{t} a(s) \mathrm{d} s} \mathfrak{L}^{d}(0)+\int_{0}^{t} \mathrm{e}^{-\int_{s}^{t} a(v) \mathrm{d} v} k(s) \mathrm{d} s+\int_{0}^{t} \mathrm{e}^{-\int_{s}^{t} a(v) \mathrm{d} v} \sigma(s) \mathrm{d} \boldsymbol{L}^{d}(s),
$$

where the matrix exponential is defined as usual. 
Proof. Define $\mathfrak{L}^{d}$ as above and calculate, for $0 \leq s \leq t \leq T$,

$$
\begin{aligned}
& -\int_{S}^{t} a(z) \mathfrak{L}^{d}(z) \mathrm{d} z \\
& =-\int_{s}^{t} a(z) \mathrm{e}^{-\int_{0}^{z} a(v) \mathrm{d} v} \mathrm{~d} z \mathfrak{L}^{d}(0)-\int_{s}^{t} a(z) \int_{0}^{z} \mathrm{e}^{-\int_{w}^{z} a(v) \mathrm{d} v} k(w) \mathrm{d} w \mathrm{~d} z \\
& -\int_{s}^{t} a(z) \int_{0}^{z} \mathrm{e}^{-\int_{w}^{z} a(v) \mathrm{d} v} \sigma(w) \mathrm{d} \boldsymbol{L}^{d}(w) \mathrm{d} z \\
& =\left[\mathrm{e}^{-\int_{0}^{t} a(v) \mathrm{d} v}-\mathrm{e}^{-\int_{0}^{s} a(v) \mathrm{d} v}\right] \mathfrak{L}^{d}(0) \\
& -\int_{s}^{t} a(z) \int_{0}^{s} \mathrm{e}^{-\int_{w}^{z} a(v) \mathrm{d} v} k(w) \mathrm{d} w \mathrm{~d} z-\int_{s}^{t} a(z) \int_{s}^{z} \mathrm{e}^{-\int_{w}^{z} a(v) \mathrm{d} v} k(w) \mathrm{d} w \mathrm{~d} z \\
& -\int_{s}^{t} a(z) \int_{0}^{s} \mathrm{e}^{-\int_{w}^{z} a(v) \mathrm{d} v} \sigma(w) \mathrm{d} \boldsymbol{L}^{d}(w) \mathrm{d} z-\int_{s}^{t} a(z) \int_{s}^{z} \mathrm{e}^{-\int_{w}^{z} a(v) \mathrm{d} v} \sigma(w) \mathrm{d} \boldsymbol{L}^{d}(w) \mathrm{d} z \\
& =\left[\mathrm{e}^{-\int_{0}^{t} a(v) \mathrm{d} v}-\mathrm{e}^{-\int_{0}^{s} a(v) \mathrm{d} v}\right] \mathfrak{L}^{d}(0) \\
& -\int_{0}^{s} \int_{s}^{t} a(z) \mathrm{e}^{-\int_{w}^{z} a(v) \mathrm{d} v} k(w) \mathrm{d} z \mathrm{~d} w-\int_{s}^{t} \int_{w}^{t} a(z) \mathrm{e}^{-\int_{w}^{z} a(v) \mathrm{d} v} k(w) \mathrm{d} z \mathrm{~d} w \\
& -\int_{0}^{s} \int_{s}^{t} a(z) \mathrm{e}^{-\int_{w}^{z} a(v) \mathrm{d} v} \sigma(w) \mathrm{d} z \mathrm{~d} \boldsymbol{L}^{d}(w)-\int_{s}^{t} \int_{w}^{t} a(z) \mathrm{e}^{-\int_{w}^{z} a(v) \mathrm{d} v} \sigma(w) \mathrm{d} z \mathrm{~d} \boldsymbol{L}^{d}(w) \\
& =\left[\mathrm{e}^{-\int_{0}^{t} a(v) \mathrm{d} v}-\mathrm{e}^{-\int_{0}^{s} a(v) \mathrm{d} v}\right] \mathfrak{L}^{d}(0) \\
& +\int_{0}^{s}\left[\mathrm{e}^{-\int_{w}^{t} a(v) \mathrm{d} v}-\mathrm{e}^{-\int_{w}^{s} a(v) \mathrm{d} v}\right] k(w) \mathrm{d} w+\int_{s}^{t}\left[\mathrm{e}^{-\int_{w}^{t} a(v) \mathrm{d} v}-I\right] k(w) \mathrm{d} w \\
& +\int_{0}^{s}\left[\mathrm{e}^{-\int_{w}^{t} a(v) \mathrm{d} v}+\mathrm{e}^{-\int_{w}^{s} a(v) \mathrm{d} v}\right] \sigma(w) \mathrm{d} \boldsymbol{L}^{d}(w)+\int_{s}^{t}\left[\mathrm{e}^{-\int_{w}^{t} a(v) \mathrm{d} v}-I\right] \sigma(w) \mathrm{d} \boldsymbol{L}^{d}(w) \\
& =\mathfrak{L}^{d}(t)-\mathfrak{L}^{d}(s)-\int_{s}^{t} \sigma(w) \mathrm{d} \boldsymbol{L}^{d}(w)-\int_{s}^{t} k(w) \mathrm{d} w .
\end{aligned}
$$

Remark 4.3. Using Hölder continuity of the MG-FLP paths (Lemma 3.1 (ii)), we can also define integration via a pathwise approach; cf. [38]. If the pathwise integral and $L^{2}$-integral both exist, they have to be equal. The next lemma is based on this fact.

Lemma 4.2. In addition to the assumptions of Definition 4.1, let the matrix $\sigma(t)$ be nonsingular for every $0 \leq t \leq T$ and $d=(d(1), \ldots, d(n))^{\top} \in\left(0, \frac{1}{2}\right)^{n}$. Furthermore, assume that $\sigma_{i j}$ and $(\sigma)_{i j}^{-1}$ are of bounded $p(j)$-variation for some $0<p(j)<1 /(1-d(j))$ for all $1 \leq i, j \leq n$ (cf. [38]). Then we have

$$
\mathrm{d} \boldsymbol{L}^{d}(t)=\left(-\sigma(t)^{-1} k(t)+\sigma(t)^{-1} a(t) \mathfrak{L}^{d}(t)\right) \mathrm{d} t+\sigma(t)^{-1} \mathrm{~d} \mathfrak{L}^{d}(t), \quad 0 \leq t \leq T .
$$

Proof. The proof is analogous to the proof of Proposition 4.1.

The prediction result follows. The proof is a combination of Theorem 4.1 and Lemma 4.2.

Theorem 4.2. (fLOUp.) For $d=(d(1), \ldots, d(n))^{\top} \in\left(0, \frac{1}{2}\right)^{n}$, take $\sigma \in \Lambda_{T}^{d}, k:[0, T] \rightarrow$ $\mathbb{R}^{n}$, and $a:[0, T] \rightarrow \mathbb{R}^{n \times n}$, where $k$ and a are componentwise locally integrable such that $\mathrm{e}^{-\int^{t} a(v) \mathrm{d} v} \sigma(\cdot) \in \Lambda_{T}^{d}$ for all $t \in[0, T]$. Further, let $\sigma(t)$ be nonsingular for every $0 \leq t \leq T$ 
and assume that $\sigma_{i j}$ and $(\sigma)_{i j}^{-1}$ are ofbounded $p(j)$-variation for some $0<p(j)<1 /(1-d(j))$ for all $1 \leq i, j \leq n$ (cf. [38]). Then we have, for $0 \leq s \leq t \leq T$ and $u \in \mathbb{R}^{n}$,

$$
\begin{aligned}
\mathbb{E}[\exp \{ & \left.\left.i\left\langle u, \mathfrak{L}^{d}(t)\right\rangle \mid \mathcal{F}_{s}\right\}\right] \\
= & \exp \left\{i\left\langle u, \mathrm{e}^{-\int_{s}^{t} a(v) \mathrm{d} v} \mathfrak{L}^{d}(s)+\int_{s}^{t} \mathrm{e}^{-\int_{w}^{t} a(v) \mathrm{d} v} k(w) \mathrm{d} w\right\rangle\right\} \\
& \times \exp \left\{i\left\langle u, \int_{0}^{s} z_{\star}^{-d}\left(\mathbf{1}_{[0, s]} z^{d}\left(h \mathbf{1}_{[s, t]}, \cdot\right), v\right) \mathrm{d} \boldsymbol{L}^{d}(v)\right\rangle+\int_{s}^{t} \psi\left(z^{d}\left(h \mathbf{1}_{[s, t]}, v\right)^{\top} u\right) \mathrm{d} v\right\} \\
= & \exp \left\{i\left\langle u, \mathrm{e}^{-\int_{s}^{t} a(v) \mathrm{d} v} \mathfrak{L}^{d}(s)+\int_{s}^{t} \mathrm{e}^{-\int_{w}^{t} a(v) \mathrm{d} v} k(w) \mathrm{d} w\right\rangle\right\} \\
& \times \exp \left\{-i\left\langle u, \int_{0}^{s} z_{\star}^{-d}\left(\mathbf{1}_{[0, s]} z^{d}\left(h \mathbf{1}_{[s, t]}, \cdot\right), v\right) \sigma(v)^{-1} k(v) \mathrm{d} v\right\rangle\right\} \\
& \times \exp \left\{\int_{0}^{s} z_{\star}^{-d}\left(\mathbf{1}_{[0, s]} z^{d}\left(h \mathbf{1}_{[s, t]}, \cdot\right), v\right) \sigma(v)^{-1} a(v) \mathfrak{L}^{d}(v) \mathrm{d} v\right\} \\
& \times \exp \left\{\int_{0}^{s} z_{\star}^{-d}\left(\mathbf{1}_{[0, s]} z^{d}\left(h \mathbf{1}_{[s, t]}, \cdot\right), v\right) \sigma(v) \mathrm{d} \mathfrak{L}^{d}(v)+\int_{s}^{t} \psi\left(z^{d}\left(h \mathbf{1}_{[s, t]}, v\right)^{\top} u\right) \mathrm{d} v\right\},
\end{aligned}
$$

where $h(\cdot)=\mathrm{e}^{-\int^{t} \cdot a(v) \mathrm{d} v} \sigma(\cdot)$.

Remark 4.4. (General SDEs.) Using the MG-FLP decomposition (3.2) and Proposition 3.9 of [37], we see that the $i$ th component of a MG-FLP is of zero-quadratic variation if $d(i) \in$ $\left(0, \frac{1}{2}\right)$. Therefore, for $d=(d(1), \ldots, d(n))^{\top} \in\left(0, \frac{1}{2}\right)^{n}$, general SDEs driven by MG-FLPs can be considered using, for example, the theory of Zähle [40], Section 5. However, as already mentioned in [16], this does not cover CIR type processes. Using the zero-quadratic variation property and the integration concept of Russo and Vallois [32], a theory similar to that of [16] can be proven (at least up to stationary solutions, where we have to address Remark 4.1). Therefore, it is interesting to calculate the prediction of transforms of MG-FLP driven integrals, which can be achieved by Fourier methods (cf. Theorem 3.7 of [18] for the univariate fBm case). An example is the next theorem which can be proven in a similar way to results of Buchmann and Klüppelberg [10] using the idea of Theorem 3.7 and Example 3.8 of [18].

Theorem 4.3. (fLCIRp.) For $d \in\left(0, \frac{1}{2}\right)$ and $a>0$, let $X=(X(t))_{t \in[0, T]}$ be the solution to the fLOUp SDE

$$
\mathrm{d} X(t)=-\frac{a}{2} X(t) \mathrm{d} t+\mathrm{d} L^{d}(t), \quad t \in[0, T], \quad X(0) \in \mathbb{R} .
$$

Assume further that $\mathbb{E}[\exp \{X(t)\}]<\infty$ for $t \in[0, T]$. Take $f(x)=\operatorname{sign}(x) x^{2} \sigma^{2} / 4$ for $x \in \mathbb{R}$ and $\sigma>0$. Define the process $Z=(Z(t))_{t \in[0, T]}$ by $Z(t)=f(X(t))$. Then, for $0 \leq s \leq t \leq T$,

$$
Z(t)-Z(s)=-a \int_{s}^{t} Z(v) \mathrm{d} v+\sigma \int_{s}^{t} \sqrt{|Z(v)|} \mathrm{d}^{\mathrm{RV}} L^{d}(v)
$$

holds with $Z(0)=f(X(0))$. Here the integral $\int_{s}^{t} \sqrt{|Z(v)|} \mathrm{d}^{\mathrm{RV}} L^{d}(v)$ is the forward integral of Definition 1 of [32]. Furthermore, for $u \in \mathbb{R}$, we have

$$
\mathbb{E}\left[\mathrm{e}^{i u Z(t)} \mid \mathcal{F}_{S}\right]=\int_{\mathbb{R}}\left(\mathbb{E}\left[\mathrm{e}^{(i \xi+1) X(t)} \mid \mathcal{F}_{S}\right] g_{+}(\xi, u)+\mathbb{E}\left[\mathrm{e}^{(i \xi-1) X(t)} \mid \mathcal{F}_{S}\right] g_{-}(\xi, u)\right) \mathrm{d} \xi
$$


with $g_{\star}(\xi, u)=(2 \pi)^{-1} \int_{\mathbb{R}_{\star}} \mathrm{e}^{-(i \xi \star 1) x+i u f(x)} \mathrm{d} x, \star \in\{+,-\}$, where $\mathbb{E}\left[\mathrm{e}^{(i \xi+1) X(t)} \mid \mathcal{F}_{s}\right]$ is given by the analytic extension of (4.1) to $\mathbb{C}$.

\section{Fractional credit models}

We shall work in the framework of the most reduced-form credit risk models in the literature. Given a finite time horizon $T^{\star}>0$, a credit market shall be described by the bivariate process $(r, H)=(r(t), H(t))_{0 \leq t \leq T^{\star}}$ on a given probability space $(\Omega, \mathcal{F}, \mathcal{Q})$ endowed with the filtration $\left(\mathcal{F}_{t}\right)_{0 \leq t \leq T^{\star}}$, which represents the market information and satisfies the usual conditions of completeness and right continuity. The process $r$ models the short rate and $H$ the default indicator, i.e.

$$
H(t)=\mathbf{1}_{\{\tau \leq t\}}, \quad 0 \leq t \leq T^{\star}
$$

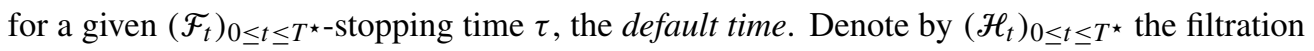
generated by $H$.

Assumption 5.1. (Market structure; cf. Assumption 3.1 of [19].)

(i) We assume that there is a subfiltration $\left(g_{t}\right)_{0 \leq t \leq T^{\star}}$ of $\left(\mathcal{F}_{t}\right)_{0 \leq t \leq T^{\star}}$ with

$$
\mathcal{F}_{t}:=\mathcal{G}_{t} \vee \mathscr{H}_{t}, \quad 0 \leq t \leq T^{\star},
$$

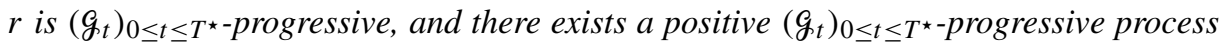
$\lambda=\left(\lambda_{t}\right)_{0 \leq t \leq T^{\star}}$, called the default rate, describing the intensity of $H$ (cf. Corollary 5.1.5 of [8]) with $\int_{0}^{t} \lambda(s) \mathrm{d} s<\infty$ a.s. for all $0 \leq t \leq T^{\star}$. Furthermore, assume that

$$
\mathbb{P}\left(\tau>t \mid g_{t}\right)=\mathbb{E}\left[1-H(t) \mid g_{t}\right]=\exp \left\{-\int_{0}^{t} \lambda(s) \mathrm{d} s\right\} .
$$

Setting $g_{\infty}:=\bigvee_{0 \leq t \leq T^{\star}} G_{t}$, assume that for all bounded $g_{\infty}$-measurable random variables $\eta$,

$$
\mathbb{E}\left[\eta \mid \mathcal{F}_{t}\right]=\mathbb{E}\left[\eta \mid g_{t}\right]
$$

holds.

(ii) $\mathcal{Q}$ is a risk neutral pricing measure, such that the price of any $\mathcal{F}_{T}$-measurable claim $X \in$ $L^{1}(\Omega)$ with maturity $0 \leq T \leq T^{\star}$ at time $0 \leq t \leq T$ is given by $\mathcal{V}(t, T)=\mathbb{E}\left[X \mid \mathcal{F}_{t}\right]$ for $0 \leq t \leq T$.

In the framework above, the default history $\left(\mathcal{F}_{t}\right)_{0 \leq t \leq T^{\star}}$ is the investor information at time $t$, meaning that the investor knows the short rate $r$, the default rate $\lambda$, and the default indicator process $H$ at time $t$. Using Lemma 13.2 of [15], we see that the price of a defaultable zero coupon bond is, for $0 \leq t \leq T \leq T^{\star}$, given by

$$
\bar{B}(t, T)=\mathbb{E}\left[\mathbf{1}_{\{\tau>T\}} \mathrm{e}^{-\int_{t}^{T} r(s) \mathrm{d} s} \mid \mathcal{F}_{t}\right]=\mathbf{1}_{\{\tau>t\}} \mathbb{E}\left[\mathrm{e}^{-\int_{t}^{T}(r(s)+\lambda(s)) \mathrm{d} s} \mid g_{t}\right] .
$$

Therefore, it is sufficient to specify the dynamics of the bivariate process $(r, \lambda)$, forgetting $H$. We propose a fractional Vasicek model.

Assumption 5.2. (Fractional Vasicek model.) For $d=(d(1), \ldots, d(n))^{\top} \in\left(0, \frac{1}{2}\right)^{n}, n \in \mathbb{N}$, take $\sigma \in \Lambda_{T^{\star}}^{d}, k:\left[0, T^{\star}\right] \rightarrow \mathbb{R}^{n}$, and $a:\left[0, T^{\star}\right] \rightarrow \mathbb{R}^{n \times n}$, where $k$ and a are componentwise locally integrable such that $\mathrm{e}^{-\int^{t} \cdot a(v) \mathrm{d} v} \sigma(\cdot) \in \Lambda_{T^{\star}}^{d}$ for all $t \in\left[0, T^{\star}\right]$. Consider the 
corresponding Ornstein-Uhlenbeck SDE

$$
\mathrm{d} \mathfrak{L}^{d}(t)=\left(k(t)-a(t) \mathfrak{L}^{d}(t)\right) \mathrm{d} t+\sigma(t) \mathrm{d} \boldsymbol{L}^{d}(t), \quad t \in\left[0, T^{\star}\right], \quad \mathfrak{L}^{d}(0) \in \mathbb{R}^{n \times n} .
$$

Further assume, as in Lemma 4.2, that $\sigma(t)$ is nonsingular for every $0 \leq t \leq T^{\star}$ and $\sigma_{i j}$ and $(\sigma)_{i j}^{-1}$ are of bounded $p(j)$-variation for some $0<p(j)<1 /(1-d(j))$ for all $1 \leq i, j \leq n$ (cf. [38]). Then define, for fixed weights $\theta, \phi \in\left(\mathbb{R}_{+}\right)^{n}$, where $\theta \neq 0$,

$$
r(t)=\left\langle\theta, \mathfrak{L}^{d}(t)\right\rangle \quad \text { and } \quad \lambda(t)=\left\langle\phi, \mathfrak{L}^{d}(t)\right\rangle, \quad t \in\left[0, T^{\star}\right] .
$$

Therefore, we have $g_{t}=\sigma \overline{\left\{\boldsymbol{L}^{d}(s), s \in[0, t]\right\}}$ for all $t \in\left[0, T^{\star}\right]$.

The following theorem considers the price of a defaultable zero coupon bond in the fractional Vasicek credit market, Assumption 5.2.

Theorem 5.1. Let $0 \leq t \leq T \leq T^{\star}$. In the model of Assumption 5.2, set $D(t, T):=$ $\int_{t}^{T} \mathrm{e}^{-\int_{t}^{s} a(v) \mathrm{d} v} \mathrm{~d}$ s where the integral is taken componentwise. Assume further that we have $D(\cdot, T) \sigma(\cdot) \in \Lambda_{T}^{d}$ and

$$
\mathbb{E}\left[\exp \left\{-\left\langle\theta+\phi, \int_{t}^{T} D(w, T) \sigma(w) \mathrm{d} \boldsymbol{L}^{d}(w)\right\rangle\right\}\right]<\infty .
$$

Then we have

$$
\begin{aligned}
\bar{B}(t, T)= & \mathbf{1}_{\{\tau>t\}} \mathbb{E}\left[\mathrm{e}^{-\int_{t}^{T}(r(s)+\lambda(s)) \mathrm{d} s} \mid g_{t}\right] \\
= & \mathbf{1}_{\{\tau>t\}} \exp \left\{-\left\langle\theta+\phi, D(t, T) \mathfrak{L}^{d}(t)+\int_{t}^{T} D(v, T) k(v) \mathrm{d} v\right\rangle\right\} \\
& \times \exp \left\{-\left\langle\theta+\phi, \int_{0}^{t} z_{\star}^{-d}\left(\mathbf{1}_{[0, t]} z^{d}\left(h \mathbf{1}_{[t, T]}, \cdot\right), v\right) \mathrm{d} \boldsymbol{L}^{d}(v)\right\rangle\right\} \\
& \times \exp \left\{\int_{t}^{T} \psi\left(z^{d}\left(h \mathbf{1}_{[t, T]}, v\right)^{\top} i(\theta+\phi)\right) \mathrm{d} v\right\},
\end{aligned}
$$

with $h(\cdot)=D(\cdot, T) \sigma(\cdot)$.

Proof. By Assumption 5.2 the stochastic integrals also exist in the pathwise sense. By the proof of Proposition 4.1 and Fubini's Theorem (pathwise), we get

$$
\begin{aligned}
\int_{t}^{T}(r(s)+\lambda(s)) \mathrm{d} s & \\
= & (\theta+\phi)^{\top} \int_{t}^{T} \mathfrak{L}^{d}(s) \mathrm{d} s \\
= & (\theta+\phi)^{\top} \int_{t}^{T}\left[\mathrm{e}^{-\int_{t}^{s} a(v) \mathrm{d} v} \mathfrak{L}^{d}(t)+\int_{t}^{s} \mathrm{e}^{-\int_{w}^{s} a(v) \mathrm{d} v} k(w) \mathrm{d} w\right. \\
& \left.\quad+\int_{t}^{s} \mathrm{e}^{-\int_{w}^{s} a(v) \mathrm{d} v} \sigma(w) \mathrm{d} \boldsymbol{L}^{d}(w)\right] \mathrm{d} s \\
= & (\theta+\phi)^{\top}\left[D(t, T) \mathfrak{L}^{d}(t)+\int_{t}^{T} D(v, T) k(v) \mathrm{d} v+\int_{t}^{T} D(w, T) \sigma(w) \mathrm{d} \boldsymbol{L}^{d}(w)\right] .
\end{aligned}
$$




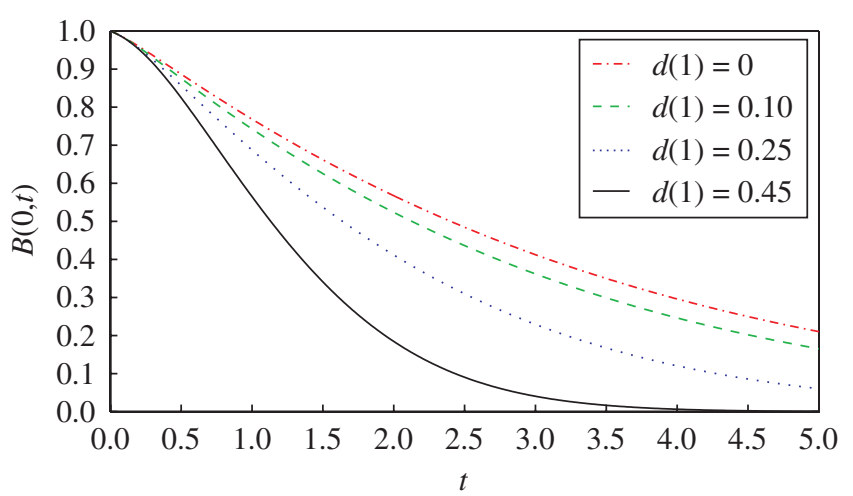

Figure 1: Bond prices $B(0, t)$ in the fractional Poisson market, Example 5.1, for varying $d(1)$ and maturity $t$, using $\left(\eta_{1}, \eta_{2}, \eta_{3}\right)^{\top}=(2,1,2)^{\top},(r(0), \lambda(0))^{\top}=(0.1,0.05)^{\top}, k_{1}=0.5, k_{2}=1, a_{1}=4$, $a_{2}=8, \sigma_{1}=2, \sigma_{2}=1$, and $d(2)=0.25$. Recall that $(d(1), d(2))^{\top}=(0,0)^{\top}$ corresponds to the Lévy Vasicek model of Remark 5.3. Prices decrease with $d(1)$ as a consequence of the long range dependence, which is very surprising; cf. Remark 5.4.

We calculate

$$
\begin{aligned}
\mathbb{E}\left[\mathrm{e}^{-\int_{t}^{T}(r(s)+\lambda(s)) \mathrm{d} s} \mid g_{t}\right]= & \exp \left\{-\left\langle\theta+\phi, D(t, T) \mathfrak{L}^{d}(t)+\int_{t}^{T} D(v, T) k(w) \mathrm{d} w\right\rangle\right\} \\
& \times \mathbb{E}\left[\exp \left\{-\left\langle\theta+\phi, \int_{t}^{T} D(w, T) \sigma(w) \mathrm{d} \boldsymbol{L}^{d}(w)\right\rangle\right\} \mid g_{t}\right] .
\end{aligned}
$$

By assumption, the conditional expectation is a.s. smaller than infinity and, therefore, we can invoke the prediction result of Theorem 4.1 (by extending it to $u \in \mathbb{C}$ ) to obtain

$$
\begin{aligned}
\mathbb{E}[\exp \{ & \left.\left.-\left\langle\theta+\phi, \int_{t}^{T} D(w, T) \sigma(w) \mathrm{d} \boldsymbol{L}^{d}(w)\right\rangle\right\} \mid g_{t}\right] \\
= & \exp \left\{-\left\langle\theta+\phi, \int_{0}^{t} z_{\star}^{-d}\left(\mathbf{1}_{[0, t]} z^{d}\left(h \mathbf{1}_{[t, T]}, \cdot\right), v\right) \mathrm{d} \boldsymbol{L}^{d}(v)\right\rangle\right\} \\
& \times \exp \left\{\int_{t}^{T} \psi\left(z^{d}\left(h \mathbf{1}_{[t, T]}, v\right)^{\top} i(\theta+\phi)\right) \mathrm{d} v\right\},
\end{aligned}
$$

with $h(\cdot)=D(\cdot, T) \sigma(\cdot)$. Putting everything together we obtain the assertion.

Remark 5.1. Condition (5.1) is met if we assume that the components of $\boldsymbol{L}^{d}$ are fractional subordinators and $\sigma(\cdot)$ is componentwise positive. These assumptions are economically justified since interest rates should be positive in most cases. We refer the reader to Theorem 3.3 of [31] for more general conditions.

Remark 5.2. The Gaussian case has already been considered in [6] and [18] using different, partly more direct, approaches. It has the serious drawback that processes like the short rate could be negative. However, in practice the Gaussian models are fast to implement and very tractable. Of course, it is always possible to scale and shift a Gaussian Vasicek model such that the probability of becoming negative is arbitrarily small. However, with the theory in this paper we can propose models with nonnegative short and default rates where bond prices are 


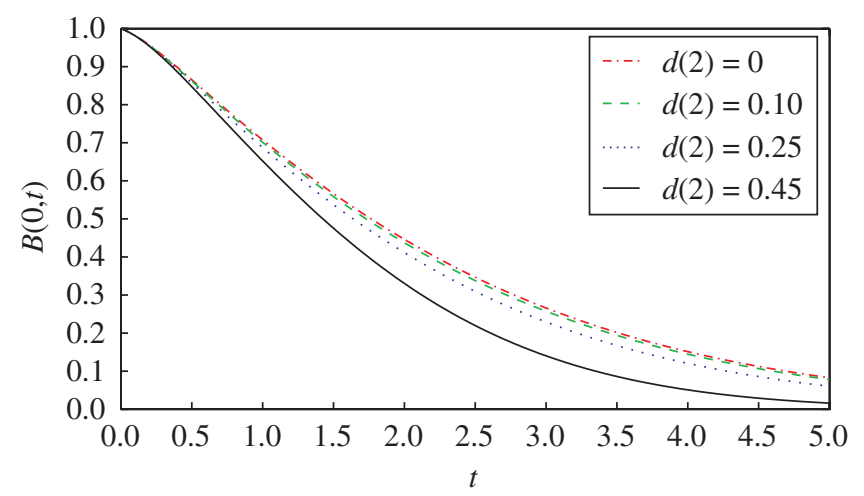

Figure 2: Bond prices $B(0, t)$ in the fractional Poisson market, Example 5.1, for varying $d(2)$ and maturity $t$, using $\left(\eta_{1}, \eta_{2}, \eta_{3}\right)^{\top}=(2,1,2)^{\top},(r(0), \lambda(0))^{\top}=(0.1,0.05)^{\top}, k_{1}=0.5, k_{2}=1, a_{1}=4$, $a_{2}=8, \sigma_{1}=2, \sigma_{2}=1$, and $d(1)=0.25$. Recall that $(d(1), d(2))^{\top}=(0,0)^{\top}$ corresponds to the Lévy Vasicek model of Remark 5.3. Prices decrease with $d(1)$ and $d(2)$ as a consequence of the long range dependence; cf. Remark 5.4.

comparably easy to calculate. On the other hand, we want to mention that, when considering credit derivatives like credit default swaps (CDS), negative interest and hazard rates might be suitable for modeling market anomalies like inverted CDS curves; cf. [17].

The remainder of this paper is dedicated to the consideration of a specific example using the bivariate Poisson MG-FLP of Example 4.3. The components of this process are fractional subordinators in the sense of Example 3.1 and, therefore, have the advantage of delivering nonnegative short and hazard rates.

Example 5.1. (Fractional Poisson market.) Assume $d(1), d(2)>0$. Take the bivariate Poisson MG-FLP of Example 4.3 as the driving process in the fractional market Assumption 5.2. Further, for simplicity, take $k(\cdot)=\left(k_{1}, k_{2}\right)^{\top}$ with $k_{1}, k_{2}>0$,

$$
a(\cdot)=\left(\begin{array}{cc}
a_{1} & 0 \\
0 & a_{2}
\end{array}\right), \quad \sigma(\cdot)=\left(\begin{array}{cc}
\sigma_{1} & 0 \\
0 & \sigma_{2}
\end{array}\right)
$$

for $a_{1}, a_{2}, \sigma_{1}, \sigma_{2}>0, \theta=(1,0)^{\top}$, and $\phi=(0,1)^{\top}$. Therefore,

$$
\begin{array}{ll}
\mathrm{d} r(t)=\left(k_{1}-a_{1} r(t)\right) \mathrm{d} t+\sigma_{1} \mathrm{~d} L^{d(1)}(t), & r(0)=r_{0} \in \mathbb{R}, \\
\mathrm{d} \lambda(t)=\left(k_{2}-a_{2} \lambda(t)\right) \mathrm{d} t+\sigma_{2} \mathrm{~d} L^{d(2)}(t), & \lambda(0)=\lambda_{0} \in \mathbb{R},
\end{array}
$$

where $L^{d(1)}$ and $L^{d(2)}$ are dependent.

We now apply Theorem 5.1. Condition (5.1) is met since the process in the exponential is nonpositive. Further, we have

$$
\begin{aligned}
D(t, T) & =\int_{t}^{T} \mathrm{e}^{-\int_{t}^{s} a(v) \mathrm{d} v} \mathrm{~d} s \\
& =\left(\begin{array}{cc}
\int_{t}^{T} \mathrm{e}^{-a_{1}(s-t)} \mathrm{d} s & 0 \\
0 & \int_{t}^{T} \mathrm{e}^{-a_{2}(s-t)} \mathrm{d} s
\end{array}\right) \\
& =:\left(\begin{array}{cc}
D_{1}(t, T) & 0 \\
0 & D_{2}(t, T)
\end{array}\right) .
\end{aligned}
$$


Therefore, we have

$$
\begin{aligned}
-\langle\theta & \left.+\phi, D(t, T) \mathfrak{L}^{d}(t)+\int_{t}^{T} D(v, T) k(v) \mathrm{d} v\right\rangle \\
& =-D_{1}(t, T) r(t)-D_{2}(t, T) \lambda(t)-k_{1} \int_{t}^{T} D_{1}(v, T) \mathrm{d} v-k_{2} \int_{t}^{T} D_{2}(v, T) \mathrm{d} v
\end{aligned}
$$

and

$$
\begin{aligned}
-\langle\theta+ & \left.\phi, \int_{0}^{t} z_{\star}^{-d}\left(\mathbf{1}_{[0, t]} z^{d}\left(h(\cdot) \mathbf{1}_{[t, T]}, \cdot\right), v\right) \mathrm{d} \boldsymbol{L}^{d}(v)\right\rangle \\
= & -\sigma_{1} \int_{0}^{t} z_{\star}^{-d}\left(\mathbf{1}_{[0, t]} z^{d}\left(D_{1}(\cdot, T) \mathbf{1}_{[t, T]}, \cdot\right), v\right) \mathrm{d} L^{d(1)}(v) \\
& -\sigma_{2} \int_{0}^{t} z_{\star}^{-d}\left(\mathbf{1}_{[0, t]} z^{d}\left(D_{2}(\cdot, T) \mathbf{1}_{[t, T]}, \cdot\right), v\right) \mathrm{d} L^{d(2)}(v) \\
= & -\int_{0}^{t} z_{\star}^{-d}\left(\mathbf{1}_{[0, t]} z^{d}\left(D_{1}(\cdot, T) \mathbf{1}_{[t, T]}, \cdot\right), v\right)\left(-k_{1} \mathrm{~d} v+a_{1} r(v) \mathrm{d} v+\mathrm{d} r(v)\right) \\
& -\int_{0}^{t} z_{\star}^{-d}\left(\mathbf{1}_{[0, t]} z^{d}\left(D_{2}(\cdot, T) \mathbf{1}_{[t, T]}, \cdot\right), v\right)\left(-k_{2} \mathrm{~d} v+a_{2} \lambda(v) \mathrm{d} v+\mathrm{d} \lambda(v)\right),
\end{aligned}
$$

where we used Lemma 4.2 in the last line. Applying the characterization of the Lévy measure from Example 4.3, we calculate

$$
\begin{aligned}
\psi\left(z^{d}\left(h \mathbf{1}_{[t, T]}, v\right)^{\top} i(\theta+\phi)\right) & \\
= & \psi\left(\left(\begin{array}{cc}
\sigma_{1} z^{d(1)}\left(D_{1}(\cdot, T) \mathbf{1}_{[t, T]}, v\right) & 0 \\
0 & \sigma_{2} z^{d(2)}\left(D_{2}(\cdot, T) \mathbf{1}_{[t, T]}, v\right)
\end{array}\right) i(\theta+\phi)\right) \\
= & \psi\left(\left(\begin{array}{c}
i \sigma_{1} z^{d(1)}\left(D_{1}(\cdot, T) \mathbf{1}_{[t, T]}, v\right) \\
i \sigma_{2} z^{d(2)}\left(D_{2}(\cdot, T) \mathbf{1}_{[t, T]}, v\right)
\end{array}\right)\right) \\
= & \eta_{1}\left(\exp \left(-\sigma_{1} z^{d(1)}\left(D_{1}(\cdot, T) \mathbf{1}_{[t, T]}, v\right)\right)-1\right) \\
& +\eta_{2}\left(\exp \left(-\sigma_{1} z^{d(1)}\left(D_{1}(\cdot, T) \mathbf{1}_{[t, T]}, v\right)-\sigma_{2} z^{d(2)}\left(D_{2}(\cdot, T) \mathbf{1}_{[t, T]}, v\right)\right)-1\right) \\
& +\eta_{3}\left(\exp \left(-\sigma_{2} z^{d(2)}\left(D_{2}(\cdot, T) \mathbf{1}_{[t, T]}, v\right)\right)-1\right) .
\end{aligned}
$$

Putting everything together and applying Theorem 5.1 leads, for all $0 \leq t \leq T \leq T^{\star}$, to the bond prices 


$$
\begin{aligned}
\bar{B}(t, T) & \\
= & \mathbf{1}_{\{\tau>t\}} \mathbb{E}\left[\mathrm{e}^{-\int_{t}^{T}(r(s)+\lambda(s)) \mathrm{d} s} \mid g_{t}\right] \\
= & \mathbf{1}_{\{\tau>t\}} \exp \left\{-D_{1}(t, T) r(t)-D_{2}(t, T) \lambda(t)\right. \\
& \left.\quad-k_{1} \int_{t}^{T} D_{1}(v, T) \mathrm{d} v-k_{2} \int_{t}^{T} D_{2}(v, T) \mathrm{d} v\right\} \\
& \times \exp \left\{-\int_{0}^{t} z_{\star}^{-d}\left(\mathbf{1}_{[0, t]} z^{d}\left(D_{1}(\cdot, T) \mathbf{1}_{[t, T]}, \cdot\right), v\right)\left(-k_{1} \mathrm{~d} v+a_{1} r(v) \mathrm{d} v+\mathrm{d} r(v)\right)\right\} \\
& \times \exp \left\{-\int_{0}^{t} z_{\star}^{-d}\left(\mathbf{1}_{[0, t]} z^{d}\left(D_{2}(\cdot, T) \mathbf{1}_{[t, T]}, \cdot\right), v\right)\left(-k_{2} \mathrm{~d} v+a_{2} \lambda(v) \mathrm{d} v+\mathrm{d} \lambda(v)\right)\right\} \\
& \times \exp \left\{\eta_{1} \int_{t}^{T}\left(\exp \left(-\sigma_{1} z^{d(1)}\left(D_{1}(\cdot, T) \mathbf{1}_{[t, T]}, v\right)\right)-1\right) \mathrm{d} v\right\} \\
& \times \exp \left\{\eta _ { 2 } \int _ { t } ^ { T } \left(\operatorname { e x p } \left(-\sigma_{1} z^{d(1)}\left(D_{1}(\cdot, T) \mathbf{1}_{[t, T]}, v\right)\right.\right.\right. \\
& \times \exp \left\{\eta_{3} \int_{t}^{T}\left(\exp \left(-\sigma_{2} z^{d(2)}\left(D_{2}(\cdot, T) \mathbf{1}_{[t, T]}, v\right)\right)-1\right) \mathrm{d} v\right\} .
\end{aligned}
$$

Remark 5.3. If we choose $d=(0,0)^{\top}$ in the context of Example 5.1 then short and hazard rates are driven by Lévy processes which are a subclass of affine Markov processes. Therefore, the bond prices can be calculated as

$$
\begin{aligned}
B(t, T)= & \exp \left\{-D_{1}(0, T) r(0)-D_{2}(0, T) \lambda(0)\right. \\
& \left.-k_{1} \int_{0}^{T} D_{1}(v, T) \mathrm{d} v-k_{2} \int_{0}^{T} D_{2}(v, T) \mathrm{d} v\right\} \\
& \times \exp \left\{\eta_{1} \int_{t}^{T}\left(\exp \left(-\sigma_{1} D_{1}(v, T)\right)-1\right) \mathrm{d} v\right. \\
& \left.+\eta_{3} \int_{t}^{T}\left(\exp \left(-\sigma_{2} D_{2}(v, T)\right)-1\right) \mathrm{d} v\right\} \\
& \times \exp \left\{\eta_{2} \int_{t}^{T}\left(\exp \left(-\sigma_{1} D_{1}(v, T)-\sigma_{2} D_{2}(v, T)\right)-1\right) \mathrm{d} v\right\},
\end{aligned}
$$

which represents the affine structure; see, e.g. [11] and [12]. However, if $d \neq(0,0)^{\top}$, the past paths of short and default rates matter and will be involved in the prices.

To compare prices we consider the case $t=0$. 
Example 5.2. In the situation of Example 5.1 we have

$$
\begin{aligned}
B(0, T)= & \exp \left\{-D_{1}(0, T) r(0)-D_{2}(0, T) \lambda(0)\right. \\
& \left.-k_{1} \int_{0}^{T} D_{1}(v, T) \mathrm{d} v-k_{2} \int_{0}^{T} D_{2}(v, T) \mathrm{d} v\right\} \\
\times & \exp \left\{\eta_{1} \int_{0}^{T}\left(\exp \left(-\sigma_{1} z^{d(1)}\left(D_{1}(\cdot, T) \mathbf{1}_{[0, T]}, v\right)\right)-1\right) \mathrm{d} v\right\} \\
\times & \exp \left\{\eta _ { 2 } \int _ { 0 } ^ { T } \left(\operatorname { e x p } \left(-\sigma_{1} z^{d(1)}\left(D_{1}(\cdot, T) \mathbf{1}_{[0, T]}, v\right)\right.\right.\right. \\
& \left.\left.\left.\quad-\sigma_{2} z^{d(2)}\left(D_{2}(\cdot, T) \mathbf{1}_{[0, T]}, v\right)\right)-1\right) \mathrm{~d} v\right\} \\
& \times \exp \left\{\eta_{3} \int_{0}^{T}\left(\exp \left(-\sigma_{2} z^{d(2)}\left(D_{2}(\cdot, T) \mathbf{1}_{[0, T]}, v\right)\right)-1\right) \mathrm{d} v\right\} .
\end{aligned}
$$

Because of the singularities in the operator $z^{d}$, classical numerical methods have to be used with care. We choose a similar discretization scheme as in the fBm case; cf. [18]. This we explain for the first occurring fractional integration.

For $d(1) \in\left(0, \frac{1}{2}\right)$ and $0 \leq t \leq T \leq T^{\star}$, we have

$$
\begin{aligned}
\int_{0}^{T} & \exp \left(-\sigma_{1} z^{d(1)}\left(D_{1}(\cdot, T) \mathbf{1}_{[0, T]}, v\right)\right) \mathrm{d} v \\
\quad= & \int_{0}^{T} \exp \left(-\sigma_{1} c_{d(1)} v^{-d(1)} \int_{v}^{T^{\star}} \frac{r^{d(1)} D(r, T) \mathbf{1}_{[0, T]}(r)}{(r-v)^{1-d(1)}} \mathrm{d} r\right) \mathrm{d} v \\
& =\sum_{i=0}^{n-1} \int_{v_{i}}^{v_{i+1}} \exp \left(-\sigma_{1} c_{d(1)} v^{-d(1)} \int_{v}^{T} \frac{r^{d(1)} D(r, T) \mathbf{1}_{[0, T]}(r)}{(r-v)^{1-d(1)}} \mathrm{d} r\right) \mathrm{d} v
\end{aligned}
$$

where the last line is obtained by decomposing the outer integral for $n \in \mathbb{N}$ and $0=v_{0} \leq v_{1} \leq$ $\cdots \leq v_{n}=T$. Now, similar to Example 4.2 of [18], for sufficiently small intervals [ $\left.v_{i}, v_{i+1}\right]$, subpartitions $v_{i}=w_{0}^{i} \leq w_{1}^{i} \leq \cdots \leq w_{m_{i}}^{i}=v_{i+1}$ for some $m_{i} \in \mathbb{N}, i=0, \ldots, n-1$, and $v \in\left[v_{i}, v_{i+1}\right]$, we have

$$
\begin{aligned}
\int_{v}^{T} \frac{r^{d(1)} D(r, T) \mathbf{1}_{[0, T]}(r)}{(r-v)^{1-d(1)} \mathrm{d} r} \approx & \frac{1}{2 d(1)} \sum_{j=0}^{m_{i}-1}\left[\left(w_{j+1}^{i}-v_{i}\right)^{d(1)}-\left(w_{j}^{i}-v_{i}\right)^{d(1)}\right] \\
& \times\left[\left(w_{j}^{i}\right)^{d(1)} D\left(w_{j}^{i}, T\right)+\left(w_{j+1}^{i}\right)^{d(1)} D\left(w_{j+1}^{i}, T\right)\right]
\end{aligned}
$$


Putting everything together we get

$$
\begin{aligned}
\int_{0}^{T} & \exp \left(-\sigma_{1} z^{d(1)}\left(D_{1}(\cdot, T) \mathbf{1}_{[0, T]}, v\right)\right) \mathrm{d} v \\
= & \sum_{i=0}^{n-1} \int_{v_{i}}^{v_{i+1}} \exp \left(-\sigma_{1} c_{d(1)} v^{-d(1)} \int_{v}^{T} \frac{r^{d(1)} D(r, T) \mathbf{1}_{[0, T]}(r)}{(r-v)^{1-d(1)}} \mathrm{d} r\right) \mathrm{d} v \\
& \approx \sum_{i=0}^{n-1} \int_{v_{i}}^{v_{i+1}} \exp \left(-\sigma_{1} c_{d(1)} A\left(v_{i}\right) v^{-d(1)}\right) \mathrm{d} v \\
& \approx \sum_{i=0}^{n-1}\left[v_{i+1}-v_{i}\right] \exp \left(-\sigma_{1} c_{d(1)} A\left(v_{i}\right)\left[v_{i+1}-v_{i}\right] / 2\right) .
\end{aligned}
$$

Choosing now $v_{i}=0.01 i, i=0, \ldots, 100 t$, and $w_{j}^{i}=0.01(i+j), j=0, \ldots, 100 t-i$, we obtain

$$
\begin{aligned}
A\left(v_{i}\right)=\sum_{j=0}^{100 t-i-1} & {\left[(j+1)^{\kappa}-j^{\kappa}\right] } \\
& \times\left[(i+j)^{\kappa} D(0.01(i+j), t)+(i+j+1)^{\kappa} D(0.01(i+j+1), t)\right]
\end{aligned}
$$

and

$$
\int_{0}^{T} \exp \left(-\sigma_{1} z^{d(1)}\left(D_{1}(\cdot, T) \mathbf{1}_{[0, T]}, v\right)\right) \mathrm{d} v \approx 0.01 \sum_{i=0}^{n-1} \exp \left(-0.005 \sigma_{1} c_{d(1)} A\left(v_{i}\right)\right) .
$$

Numerical examples can be found in Figures 1 and 2.

Remark 5.4. At first sight it is surprising that, in the case of a fractional Poisson market, prices decrease with $d(1)$ and $d(2)$, since the contrary happens in the Gaussian case (cf. [18]). The reason behind this is the following: in a fractional Poisson market, short and default rates increase with the shocks of the driving Poisson subordinators and decrease between these shocks exponentially. An increase in $d(1)$ and $d(2)$ means an increase in the (positive) correlation between these shocks. Therefore, short and default rates are more likely to go up, which leads the bond price to drop.

In the Gaussian case, the driving processes are not increasing and an increase in $d(1)$ and $d(2)$ no longer affects short and default rates, as above, since there is now also a (positive) correlation between decreases of the driving processes.

\section{Acknowledgements}

This work was completed while the author visited the Oxford-Man Institute of Quantitative Finance. He gratefully acknowledges the pleasant hospitality. Furthermore, the author wants to thank Christian Bender and an anonymous referee for their useful comments which led to a real improvement of the paper.

\section{References}

[1] Applebaum, D. (2004). Lévy Processes and Stochastic Calculus. Cambridge University Press. 
[2] Benassi, A., Cohen, S. And Istas, J. (2002). Identification and properties of real harmonizable fractional Lévy motions. Bernoulli 8, 97-115.

[3] Bender, C. And Elliott, R. J. (2003). On the Clark-Ocone theorem for fractional Brownian motions with Hurst parameter bigger than a half. Stoch. Stoch. Rep. 75, 391-405.

[4] Bender, C. And Marquardt, T. (2008). Stochastic calculus for convoluted Lévy processes. Bernoulli 14, 499-518.

[5] Bender, C. And Marquardt, T. (2009). Integrating volatility clustering into exponential Lévy models. J. Appl. Prob. 46, 609-628.

[6] Biagini, F., Fink, H. ANd KlüpPelberg, C. (2013). A fractional credit model with long range dependent default rate. Stoch. Process. Appl. 123, 1319-1347.

[7] Biagini, F., Fuschini, S. And KlÜPpelberg, C. (2011). Credit contagion in a long range dependent macroeconomic factor model. In Advanced Mathematical Methods in Finance, eds G. Di Nunno and B. Øksendal, Springer, Heidelberg, pp. 105-132.

[8] Bielecki, T. R. ANd RutKowski, M. (2002). Credit Risk: Modelling, Valuation and Hedging. Springer, Berlin.

[9] Blumenthal, R. M. and Getoor, R. K. (1961). Sample functions of stochastic processes with stationary independent increments. J. Math. Mech. 10, 493-516.

[10] Buchmann, B. And KLÜPPELBERG, C. (2006). Fractional integral equations and state space transforms. Bernoulli 12, 431-456.

[11] Duffie, D. (2004). Credit risk modeling with affine processes. 2002 Cattedra Galileana Lecture, Stanford University and Scuola Normale Superiore, Pisa.

[12] Duffie, D., Filipović, D. And Schachermayer, W. (2003). Affine processes and applications in finance. Ann. Appl. Prob. 13, 984-1053.

[13] Duncan, T. E. (2006). Prediction for some processes related to a fractional Brownian motion. Statist. Prob. Lett. 76, 128-134.

[14] Elliott, R. J. AND VAn DER Hoek, J. (2003). A general fractional white noise theory and applications to finance. Math. Finance 13, 301-330.

[15] Filipovic, D. (2003). Term-Structure Models. Springer, New York.

[16] FinK, H. AND KlÜPPelberG, C. (2011). Fractional Lévy-driven Ornstein-Uhlenbeck processes and stochastic differential equations. Bernoulli 17, 484-506.

[17] FINK, H. AND SCHERR, C. (2012). Credit risk with long memory. Submitted.

[18] Fink, H., KLÜPPElberg, C. ANd Z̈̈HLE, M. (2013). Conditional distributions of processes related to fractional Brownian motion. J. Appl. Prob. 50, 166-183.

[19] Frey, R. And Backhaus, J. (2008). Pricing and hedging of portfolio credit derivatives with interacting default intensities. Internat. J. Theoret. Appl. Finance 11, 611-634.

[20] Henry, M. And ZafFaroni, P. (2003). The long-range dependence paradigm for macroeconomics and finance. In Theory and Applications of Long-Range Dependence, eds P. Doukhan, G. Oppenheim, and M. Taqqu, Birkhäuser, Boston, MA, pp. 417-438.

[21] Ikeda, N. and Watanabe, S. (1989). Stochastic Differential Equations and Diffusion Processes, 2nd edn. North-Holland, Amsterdam.

[22] Jost, C. (2006). Transformation formulas for fractional Brownian motion. Stoch. Process. Appl. 116, 1341-1357.

[23] KlüPPElberg, C. AND Matsui, M. (2010). Generalized fractional Lévy processes with fractional Brownian motion limit. Submitted. Available at www-m4.ma.tum.de/Papers.

[24] Marcus, M. B. ANd Rosiński, J. (2005). Continuity and boundedness of infinitely divisible processes: a Poisson point process approach. J. Theoret. Prob. 4, 109-160.

[25] Marquardt, T. (2006). Fractional Lévy processes with an application to long memory moving average processes. Bernoulli 12, 1099-1126.

[26] Marquardt, T. (2007). Multivariate fractionally integrated CARMA processes. J. Multivariate Analysis 98, 1705-1725.

[27] Monroe, I. (1972). On the $\gamma$-variation of processes with stationary independent increments. Ann. Math. Statist. 43, 1213-1220.

[28] Ohashi, A. (2009). Fractional term structure models: no-arbitrage and consistency. Ann. Appl. Prob. 19, 15531580.

[29] Pipiras, V. and TaqQu, M. S. (2000). Integration questions related to fractional Brownian motion. Prob. Theory Relat. Fields 118, 251-291.

[30] Pipiras, V. ANd TaqQu, M. S. (2001). Are classes of deterministic integrands for fractional Brownian motion on an interval complete? Bernoulli 7, 873-897.

[31] Rajput, B. S. AND Rosiński, J. (1989). Spectral representations of infinitely divisible processes. Prob. Theory Relat. Fields 82, 451-487. 
[32] Russo, F. And Vallois, P. (2007). Elements of stochastic calculus via regularisation. In Séminaire de Probabilités $X L$ (Lecture Notes Math. 1899), Springer, Berlin, pp. 147-185.

[33] Samko, S. G., Kilbas, A. A. And Marichev, O. I. (1993). Fractional Integrals and Derivatives. Theory and Applications. Gordon and Breach, Yverdon.

[34] Samorodnitsky, G. and Taqqu, M. S. (1994). Stable Non-Gaussian Random Processes. Chapman \& Hall, New York.

[35] Sato, K.-I. (1999). Lévy Processes and Infinitely Divisible Distributions. Cambridge University Press.

[36] Sato, K.-I. (2006). Additive processes and stochastic integrals. Illinois J. Math. 50, 825-851.

[37] TikanmäKi, H. J. And Mishura, Y. (2011). Fractional Lévy processes as a result of compact interval integral transformation. Stoch. Anal. Appl. 29, 1081-1101.

[38] Young, L. C. (1936). An inequality of the Hölder type, connected with Stieltjes integration. Acta Math. 67, 251-282.

[39] Z̈̈HLE, M. (1998). Integration with respect to fractal functions and stochastic calculus. I. Prob. Theory Relat. Fields 111, 333-374.

[40] Z̈̈HLE, M. (2001). Integration with respect to fractal functions and stochastic calculus. II. Math. Nachr. 225, 145-183. 